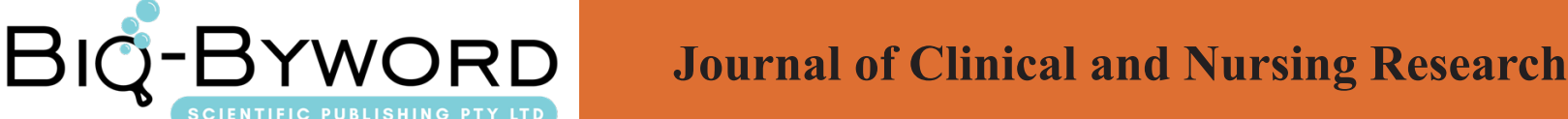

\section{Drug resistance mechanism of Staphylococcus aureus bacterial biofilm}

\author{
Tan Jia Liang
}

Benxi Shanda Hospital Liaoning Benxi 117000

\section{ARTICLE INFO}

Article history:

Published online: 15th July, 2017

Key words:

Golden yellow; Staphylococcus;

bacterial biofilm; drug resistance

mechanism

*Corresponding Author

Fu Ning, Hanzhong City, Shaanxi Province, Hantai District, North District, No. 251, Hanzhong City People's Hospital. Email address: funing2018.163com.

\begin{abstract}
Bacterial biofilm $(\mathrm{BF})$ is a kind of growth way that bacteria are associated with planktonic cells in the process of growing on the body tissue or biomaterial surface to adapt to the change of living environment, composed of bacteria and extracellular matrix. After the formation of biofilm, bacteria are coated in the extracellular matrix, increased immune system of the body and antibiotic resistance, making these two mechanisms are difficult to effectively remove the infected foci, and biofilm bacteria can also continue to grow and release into blood, resulting in chronic infection and withdrawal of antibiotics after the recurrence of infection, leading to disease progression. Based on this, this paper will focus on the analysis of Staphylococcus aureus bacterial biofilm resistance mechanism, with a view to the future practical work to play a role in reference.
\end{abstract}

\section{Introduction}

Staphylococcus aureus (SA, S. aureus) is an important pathogen in medicine, widely distributed in hospital environment, strong pathogenicity, can cause skin and mucous membranes and soft tissue abscess, otitis media, arthritis, pneumonia and other clinical common of the infection, can also cause osteomyelitis, endocarditis, meningitis and bacteremia and other serious infections. The bacteria have a strong ability to adapt to environmental factors, it is easy to obtain resistance to antibiotics and disinfectant resistance, so can survive in a hospital environment for a long time, and it is the most common pathogens in hospital infection.

\section{Staphylococcus aureus bacterial biofilm overview}

Studies had shown that formation of Staphylococcus aureus biofilm is a dynamic process, from the initial bacterial colonization to later colonization, adhesion, aggregation, differentiation and maturation until the final spread through five stages with different characteristics. The bacteria first adhere to the surface of the organism or the polymer, and then the cells become aggregated to form bacterial clumps, with the gradual increase of bacteria to form a relatively mature and stable biofilm structure, and finally, some of the bacteria in the biofilm leave and spread to the distance, which started another biofilm formation process. Biofilm formation is a process of multi-gene 
regulation and multi-factor participation, and is influenced by environmental factors. It is the result of the combined effect of each factor, and they can interact with each other to form a complex regulatory network. So far, the mechanism of biofilm formation had shown that different genes play different roles in different stages of biofilm formation. Therefore, the study of biofilm formation is an important basis for the mechanism of biofilm formation. The alpha-toxin produced by Staphylococcus aureus is a secreted protein of about $33 \mathrm{kDa}$, which is a major pathogenic molecule. Alpha-toxins interact with the target cells, the polymerization of the heptamer in the cell membrane on the formation of the transmembrane pore, causing calcium ion transmembrane flow leading to the target cell lysis. Studies had shown that the alpha-toxin-encoding gene hla was absent from Staphylococcus aureus biofilm formation in the intercellular adhesion, the aggregation of bacteria occurred and could not form a stable biofilm structure. In recent years, there had been many studies on the mechanism of biofilm formation involving alpha-toxin, but its expression in the process of biofilm formation has not been reported.

\section{Staphylococcus aureus bacterial biofilm resistance mechanism}

Staphylococcus aureus is very resistant to the clinical antibiotic. The main resistance mechanisms are: produce passivation enzymes, target modification, changes in permeability, efflux pump system and biofilm formation. Its resistance can be propagated through the bacteria reproduction and cloning; also through the plasmid, transposon transformation and transduction, spread in the level between bacteria. A bacteria can be obtained through a variety of ways and integration of multiple drug resistance genes, manifested as multiple drug resistance; can also be resistance to only one type of antibiotic.

\section{1. $\beta$-lactam}

MecA as SA-specific drug-resistant genes, resulting in SA resistance to $\beta$-lactam drugs. A penicillin-binding protein (PBPs) synthesized on the surface of bacterial cells plays an important role in the growth and reproduction of bacteria. As $\beta$-lactam antibiotics have a higher affinity with PBP, resulting in loss of activity of PBPs, blocking the synthesis of cell walls, leading to cell death. The mecA gene presents in a unique, mobile chromosome cassette, SCCmec, which encodes a novel penicillin-binding protein, PBP2a, which has a low affinity for the beta-lactam, but participates in the synthesis of cell walls as well as PBPs. Therefore, PBPs inactivated after its replacement to promote cell wall synthesis, and then showed resistance.

\subsection{Macrolides}

The main reason for the resistance of SA to macrolide is related to the erm gene located on plasmids and chromosomes, especially the ermA, ermB and ermC genes. Macrolide antibacterial drugs mainly act on the ribosomal 23S rRNA gene, blocking the synthesis of protein, play an antibacterial effect. And the drug resistance is caused by the methyltransferase erm family of 23SrRNA methylation, resulting in macrolide drugs and RNA affinity decreased or lost, resulting in drug resistance. In addition, mutation of ribosomal protein L4, mutation of 23SrRNA base of ribosomal large subunit can also cause drug resistance to macrolide.

\subsection{Fluoroquinolones (FQNS)}

The resistance of SA to FQNS is mainly related to the change of target enzyme and decrease the amount of drug that accumulates in bacteria. The chemical structure of FQNSwere1, 4-dihydro-4-oxo-3-quinolinecarboxylic acid, which has an effect on bacterial DNA gyrase (type topoisomerase) and topoisomerase inhibition, blocking bacterial inheritance substance replication and transcription, so that the bacteria die.

\subsection{Cycloaliphatic peptide}

The mechanism of bacterial resistance to cycloaliphatic peptide is mainly related to the changes of bacterial cell membrane structure and function, gene mutation and abnormal cell wall. Cycloaliphatic peptide has a role of interference to the amino acids transporter on the cell membrane, inhibit the bacterial cell wall peptidoglycan phosphate (LTA) biosynthesis, the bacterial cell membrane depolarization, causing bacterial death. The mechanisms of bacterial resistance to cycloaliphatic peptide are due to mutation of the gene in which the antibacterial target is located, resulting in a decrease in number of drug-to-bacterial connections, resulting in decreasing the ability of the drug to kill SA and drug resistance ; on the other hand, the bacteria involved in the cell wall metabolism of several genes up-regulation, making the bacterial cell wall thickening, and thus the effect of drugs on the bacteria decreased and results of drug resistance.

\subsection{Glycopeptides}

Vancomycin and other glycopeptide drug resistance mechanism, including cell wall thickening, peptidoglycan cross-linking decreased, penicillin-binding protein PBPs changes, antibiotic induction, regulation of gene changes, drug resistance gene transfer, decreased enzyme activity. The combination of the D-alanyl-D-alanine residue that presents on the cell wall peptidoglycan layer with vancomycin can block most of the drug out 
of the cell wall and reduce the lethality of the bacterium; peptidoglycan the glutamate residues on the monomeric pentapeptide branch were not amidated, resulting in a decrease in the cross-linking of the peptidoglycan, an abnormally increasing peptidoglycan monomer, whereby more vancomycin can be combined, therefore, the drug cannot bind to the target of bacteria. Drug resistance of bacteria and regulatory genes should have some certain relationship, including the function of agr gene loss; antibacterial drugs that act on cell wall such as vancomycin can increase PBP2 expression, the cell wall would be thickening, weaken the drug on killing of bacteria; test showed that vancomycin-resistant strains grew in vancomycin-free culture, bacterial resistance decreased, and thus the induction of antibiotics is also an important drug resistance factor.

\section{Staphylococcus aureus bacterial biofilm regulation mechanism}

The gene regulatory system Agr (Accessory gene regulator) group induction (Quorum-sensing) is an important mechanism to monitor the number and density of Staphylococcus aureus and also monitor surrounding environment through the specific expression genes of the group to detect any changes and to regulate the bacterial behavior. Agr is the most widely studied and well-developed population sensing system, which has great significance in bacterial colonization, invasion, biofilm formation and spread.

Agr system regulated downstream gene expression by two similar two-element proteins (Agr C and Agr A) and two reaction biofilm structures with mature proteins (Agr D and Agr B). The extracellular signal molecule Agr D was modified by Agr B as a self-inducing peptide (AIP) and expelled from bacteria. With the increasing of bacterial density and reaching a certain threshold, AIP triggered the phosphorylation of histidine kinase Agr $\mathrm{C}$, which resulting in effector molecules Agr A phosphorylation and activation; Agr A could bind to the corresponding sites of bacterial DNA regulation of downstream gene expression: to promote PSM $\alpha$ and PSM $\beta$ transcription, reduced the expression of bacterial surface protein gene level. Reduction of the bacterial surface protein caused decreased the adhesion between the bacteria, coupled with the dissociation of PSMs, resulting in biofilm bacteria spread, repeated infection cycle.

When Agr gene loss, Staphylococcus aureus biofilm would become thicker, the micropores would be reduced, the surface would be smoother and lose dissociation ability, forming a broad and dense biofilm, and the bacteria would tend to cause local chronic biofilm related infection. On the contrary, when the Agr gene overexpression, biofilm was also easy to spread quickly, more likely to cause acute infection.

The auxiliary regulator Sar A Staphylococcus aureus there was another biofilm global regulatory factor Sar A (Staphylococcalotic ATPA), the biofilm had a positive regulatory role. Sar A was encoded by sAr, which regulated the expression of nearly 120 genes in Staphylococcus aureus and promoted adhesion and early biofilm formation by up-regulating the expression of CWA in logarithmic phase and synergistically inhibiting extracellular protease production with another important gene sAeRS. In the latter part of the logarithm, SarA could be combined with its own promoter for negative feedback, contributed to the release of biofilm dissociation. SarA was also involved in the synthesis and regulation of Staphylococcus aureus PIA, which was facilitated by direct binding to the promoter of icA. Recent studies had found that there was a reduction in the ability to remove SarA bacteria biofilm formation, and increased susceptibility to antibiotics. Therefore, SarA was a potential target for the treatment of biofilm-related infections, and there were some biofilm inhibitors designed for this molecule, and there were some effects on it.

The impact of environmental factors on the biofilm, Staphylococcus aureus able to detect number and density of bacteria then make some modifications, but also able to give respond according to the changes in the surrounding environmental factors. The liquid shear force was an environmental factor that was studied at present. Under the condition of low shear force, Staphylococcus aureus formed non-colonized biofilm morphology. The rate of bacterial propagation was slow, the virulence was decreased and it was sensitive to oxidative stress. Easy to be cleared. On the contrary, under high shear conditions, biofilm, although the thickness was not large but denser, making the resistance increased. Under the condition of hypoxia, the two-component system of Staphylococcus aureus was activated, which could make the bacteria adapt to the environment and improve the expression level of PIA. The hypoxic environment could induce ciD transcription, increase eDNA content and finally promote biofilm formation. Recent studies had found that, in the case of low $\mathrm{pH}$, Staphylococcus aureus could recover cytoplasmic proteins and reversibly bind to the bacterial surface as a biofilm matrix component, enhancing the flexibility and adaptability of bacterial biofilm formation.

In short, Staphylococcus aureus is an important pathogen in medicine, widely distributed in the hospital environment, and it has strong pathogenicity, can cause skin and mucous membranes and soft tissue abscess, otitis media, arthritis, pneumonia and other clinical common 
infection, also can cause osteomyelitis, endocarditis, meningitis and bacteremia and other serious infections. The bacteria have a strong ability to adapt to environmental factors, it is easy to obtain resistance to antibiotics and resistance to disinfectants, so long-term survival in the hospital, it is one of the most common pathogens of hospital infection, so must study more and do more research about it.

\section{References}

[1] Meng J. Biological material bacterial adhesion in the role of bacterial biofilm [D]. Kunming Medical College, 2003.

[2] Xu Y. Chronic wound bacterial biofilm clinical identification and intervention of the preliminary study [D]. Nanjing University of Traditional Chinese Medicine, 2015
[3] Huang Q. Human defensin -3 on Staphylococcus aureus biofilm effect of experimental study [D]. Third Military Medical University, 2014

[4] Zhang W. Lemon extract on multi-drug resistant Staphylococcus aureus bacteriostasis and antibacterial mechanism [D]. Tianjin Medical University, 2008

[5] Li Y. Study on the biofilm formation and its influencing factors of dairy cow milk infants in Xinjiang southern Xinjiang [D]. Tarim University, 2013

[6] Wang Y. Lianhua Qingwen capsule on respiratory tract infection-related bacterial biofilm inhibition and its genetic mechanism [D]. Jilin University, 2014

[7] Han X., Wang Y., Wang Y., Li D., Liu C., Zhang T., Wang Y., Liu J., Hao Y. Effects of Qingkailing Injection on Staphylococcus aureus Bacterial Biofilm [J]. Beijing Journal of Traditional Chinese Medicine, 2014, 05: 300-303 